P. C. H. Martens, S. Tsuruta, and M. A. Weber, eds.

\title{
Summary of Posters on Solar Physics
}

\author{
M. J. Aschwanden \\ Lockheed Martin Advanced Technology Center, Solar and Astrophysics \\ Lab., Dept.L9-41, Bldg.252, 3251 Hanover St., Palo Alto, CA 94304, \\ U.S.A.
}

Five posters in the group of solar physics have been presented at this meeting on "Highly Energetic Physical Processes and Mechanisms for Emissions from Astrophysical Plasmas". Four of these posters were dealing with aspects of solar flares, the most energetic processes observed in the solar corona, while one poster dealt with active regions. Although the most energetic particles in solar flares $\left(E<10^{8} \mathrm{eV}\right)$ do not compete with the most energetic cosmic rays $\left(E>10^{18.5} \mathrm{eV}\right)$ observed in the rest of the universe, similar particle-acceleration mechanisms (electric fields, stochastic acceleration, shocks) are investigated for both phenomena. On the other hand, some highly efficient acceleration mechanisms operating in astrophysical plasmas (e.g., $E \times B$ drift in rapidly rotating B-fields on pulsars) are definitely not applicable to solar flares. However, a common theme in both astrophysical and solar acceleration processes is the role of magnetic reconnection, which was also the subject in one or the other form in all of the five presented solar posters. The joint venture of astrophysical and solar participants of this workshop contributed therefore nicely to a cross-fertilization of these common physical themes in both disciplines.

Let us briefly pass the five solar posters to remind us about their links to the main theme of this symposium:

The poster by Masuda reports on new observations of solar flares with above-the-loop-top hard X-ray sources. The discovery of these coronal hard $\mathrm{X}$-ray sources (made by Masuda in 1994) provided for the first time a direct localization of the primary acceleration site in solar flares, a key finding that was also independently corroborated by electron time-of-flight measurements (with the Compton Gamma Ray Observatory in 1995). In his poster, Masuda shows two flares that occurred a few hours apart, with and without footpoint occultation at the solar limb, so that the relative contrast between footpoint and above-the-loop-top hard X-ray sources varies greatly and, in this way, explains their detection and nondetection in the two cases.

In their poster, Uchida et al. present an application of his quadrupolar magnetic reconnection model (1980) to Yohkoh observations, illustrated and quantitatively supported with numerical MHD simulations. This model envisions $\mathrm{X}$-type reconnection between a small-scale arcade with an overarching, magnetically oppositely-oriented, large-scale arcade, driven by current injection along the central neutral filament. Uchida's model offers a solution of the energy paradox of the classical (opening-up and reclosing arcade) reconnection model and explains the basic features of the dark filaments. 
In their poster, Yokoyama \& Shibata show two-dimensional MHD simulations of the widely-accepted Y-type (Kopp \& Pneuman) reconnection model. The numeric code also includes new features such as anisotropic heat conduction. The authors vary the plasma-beta parameter in their simulations and find a scaling law between the flare temperature and magnetic field. Moreover, the authors suggest that this scaling law can be extended from solar flares to stellar flares (V773 Tauri, T-Tauri stars, etc.), and thus may have universal validity.

In another poster, Aschwanden et al. deal with the electron kinematics in reconnection-driven flares, basically employing the same flare model as simulated by Yokoyama \& Shibata in their poster and supported by Masuda's observations, presented in his poster. Given the coronal location of the acceleration site above the reconnected flare loops seen in soft X-rays (after they have been filled with heated plasma upflowing from the chromosphere), the kinematics of accelerated electrons can be reconstructed from the magnetic field geometry and pitch-angle distribution of the accelerated electrons. This poster presents new results from asymmetric flare loops, where the relative flux ratio of the conjugate footpoints and the energy-dependent time delays of HXR emission is deconvolved to determine the fraction of trapped electrons and the magnetic mirror ratios between the acceleration site and the chromospheric flare loop footpoints.

The poster by Welsch \& Longcope, although applied to active regions, deals also with general aspects of magnetic reconnection processes that are relevant in flares, as well. The authors study the role of separator boundaries in quadrupolar reconnection geometries, which are thought to dissipate magnetic energy when magnetic flux is exchanged from one topological domain to the other. A simple, analytical model is presented which quantifies the statistical amount of transferred (or dissipated) energy as function of the geometrical parameters of the quadrupolar active region. With this model, theoretical predictions of scaling laws and statistical properties of magnetic separators can be calculated. 